of these have been discussed recently in News and Views". The main focus at present is to obtain clues as to how the genes interact by studying the effects of mutations in one gene on the expression of another. With so many genes apparently involved, however, the interpretation of such experiments is unlikely to be simple, and it was notable at the meeting that most of the experimentalists averred that they found Meinhardt's model a helpful framework in which to try to interpret their results. The film Meinhardt showed at the meeting of computer simulations based on his model demonstrated that it can closely simulate the evolving patterns of segmentation gene expression in wild-type and normal embryos that are observed experimentally.

\section{Dorsal-ventral axis}

A further set of maternal-effect mutations affects pattern-formation along the dorsal-ventral axis of the Drosophila embryo. Recessive mutations in at least 10 genes cause dorsalization of the embryo, and a series of experiments ${ }^{7.8}$ reviewed recently in News and Views" has led to a model for the establishment of dorsalventral positional information by a morphogenetic product generated by these genes. One of this set, snake, has been cloned and sequenced, and encodes a protein with all the features of a serine protease (R. Delotto, University of Geneva). This is an exciting result, for serine proteases are made as inactive precursors activated by specific proteolytic cleavage, and in blood clotting and complement fixation chains of specific serine proteases are arranged in a cascade to amplify an initial signal in such a way as can readily be envisaged to be applied to the more glamorous problem of pattern formation. Both blood clotting and complement fixation generate products tightly localized in space close to the initial signal: the intriguing prospect is that such a signal, probably at the ventral pole, initiates a cascade of reactions which converts it into a set of coordinates along the dorsal-ventral axis. If this is true, other dorsalization genes should turn out to encode serine proteases.

Although the issue has been controversial, it is now generally accepted that during development the anterior-posterior axis of the Drosophila embryo is divided into polyclonal units called compartments, defined by the expression of sets of 'specifier' genes that determine their pathways of development. Whether the dorsal-ventral axis is divided into compartments analogous to those along the anterior-posterior axis is unclear. That this may be the case is suggested by recent work on another gene of the Antennapedia complex that has recently been cloned, zerknullt (zen) ${ }^{\text {I"'. Mutations }}$ of this gene affect the differentiation of

\title{
A bacterial haemoglobin
}

\section{from M.F. Perutz}

Haemoglobin was believed to be a protein that evolved in eukaryotes, but a report elsewhere in this issue (Wakabayashi, S., Matsubara, H. \& Webster, D.A. Nature 322,481 ; 1986) describes a bacterial haem protein from Vitreoscilla that combines with molecular oxygen and has an amino-acid sequence with features characteristic of the globins.

There are only two amino-acid residues that are common to all the globins: a histidine that forms a covalent link with the haem iron; and a phenylalanine in position 1 of the loop made by helices $\mathrm{C}$ and $D$ which wedges the haem into its pocket. All other residues are replaceable, but in $\mathbf{3 3}$ specific positions replacements are restricted to non-polar residues (Perutz, M.F., Kendrew, J.C. \& Watson, H.C. J.molec. Biol. 13, 669; 1965).

The haem protein of Vitreoscilla does contain non-polar residues at almost all these positions, and it shows marked homology with the haemoglobin of the leguminous nodules of lupins, whose globin is encoded by a gene in the plant, rather than the symbiotic bacterium $R h i$ zobium where the haemoglobin is concentrated. Most haemoglobins contain a histidine in a position distal to the haem, but the $\alpha$-chain of the oppossum is an exception and contains a glutamine in-

dorsally derived embryonic tissues. Analysis of the cloned gene shows that it contains a homoeo box and is expressed in dorsal tissues of developing embryos. The evolution of the pattern of zen expression along the dorsal-ventral axis during development is reminiscent of genes such as Ultrabithorax that are expressed in a compartment-specific manner along the anterior-posterior axis, suggesting that zen may be involved in the specification of a dorsal-ventral compartment.

\section{A Cartesian coordinate frame?}

Meinhardt has suggested that the points on the surface on an insect embryo where dorsal-ventral and anterior-posterior compartment boundaries intersect might specify the positions of the imaginal disks that later in development generate the adult cuticle structures. Intriguing evidence that this could be true was reported by W. Gelbert (Harvard). Mutations of the decapentaplegic gene of Drosophila cause the loss of pattern elements derived from the centres of imaginal disks. Gelbert explained that clonal analysis shows that a normal decapentaplegic gene is required only in cells that abut the anterior-posterior compartment boundary of a disk for normal development. stead. The alignment of the Vitreoscilla sequence by Wakabayashi et al. suggests that it also has a distal glutamine whose amino group could form a hydrogen bond with the bound oxygen, just as the distal histidine does in myoglobin (Phillips, S.E.V. \& Schoenborn, B. Nature 292, 81; 1981). Homology between Vitreoscilla and other globins extends to helices B, C, E, F, G and H; helix $\mathrm{D}$ is missing as it is in the $\alpha$-chains of mammalian haemoglobins, and homology ends at the A-B corner. The first 11 residues show no homology with the $A$ helix, but this helix is unimportant because it is not in contact with the haem.

What is the function of this haemoglobin? The haemoglobin content of Vitreoscilla increases almost $\mathbf{5 0}$-fold when the oxygen concentration of the growth medium falls below 10 per cent of atmospheric. Apparently, species of this genus live in oxygen-poor environments; possession of the haemoglobin helps them to absorb oxygen for their aerobic metabolism. Is this an example of divergent or convergent evolution or of gene transfer from eukaryotes? Who can guess? We were not there when it happened.

M.F. Perutz is at the Laboratory of Molecular Biology, Hills Road, Cambridge CB2 $2 Q H$, UK.

Furthermore, using the cloned gene it has been found that decapentaplegic is expressed in a dorsal region of the embryo. later splitting into two stripes running along the anterior-posterior axis on either side of the embryo just where the two lines of imaginal disks later develop. So it could be that these stripes define dorsal-ventral compartment boundaries, and the spatial cue for disk formation is where they cross anterior-posterior compartment boundaries. I am sure that the founder of analytical geometry, René Descartes, would be pleased by this elegant application of his system of spatial coordinates to biological pattern formation.

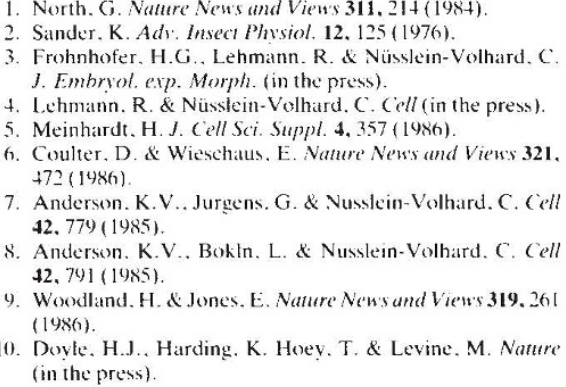

Andersion. K.V., Bokln. L. \& Nusslein-Volhard. C. Cell $42,741(1985)$

Woodland. H. \& Jones. E. Nature Nens and Viens 319.261 (1986)

0. Doyle. H.J.. Harding. K. Hoey. T. \& Levine. M. Noture (in the press)

Geoffrey North is Deputy Biology Editor of Nature. 